\title{
Analysis and Detection of Skin Cancer
}

\author{
${ }^{1}$ Sonali Jadhav, ${ }^{2}$ D. K. Kamat \\ ${ }^{I}$ Dept of $E$ \& TC,Sinhgad Academy of Engg, Pune \\ ${ }^{2}$ Asst Prof, Dept of E \& TC, Sinhgad Academy of Engg, Pune
}

\begin{abstract}
Medical image preprocessing and segmentation are very important in medical area. This paper deal with the implementation of simple algorithm for detection of skin cancer also and infected area. Skin cancer are the most common cancer in human. Skin cancer are curable cancer after early detection. In hospital doctor used a biopsy (a laboratory medical procedure). There are many different type of cancer. First step is preprocessing by using median filter for removing noise. In second step segmentation done by Thresholding and Fuzzy CMeans (FCM). Feature extraction done by Gray Level Co-Occurance Matricx (GLCM). Four different statistical features are extractd. also calculating standarad deviation of feature extract.
\end{abstract}

Index Terms: Skin cancer, Segmentation, Fuzzy c-means, GLCM.

\section{Introduction}

Skin protects body from infection. It also protects body from damage caused by ultraviolet (UV) radiation. skin stores fat and water. The figure 1 shows the two main layers of the skin:

- Epidermis: The epidermis is the first layer of our skin.It is made by flat cells. That called squamous cells.Basal Cell is bellow the squamous cells.In basal cell occure melanocytes. Melanocytes make the color found in skin.

- Dermis: The dermis is the layer inside the epidermis.

The dermis contains different types of cells and structures, blood vessels, glands, and lymph vessels.Because of these glands form sweat, which helps body cooling.
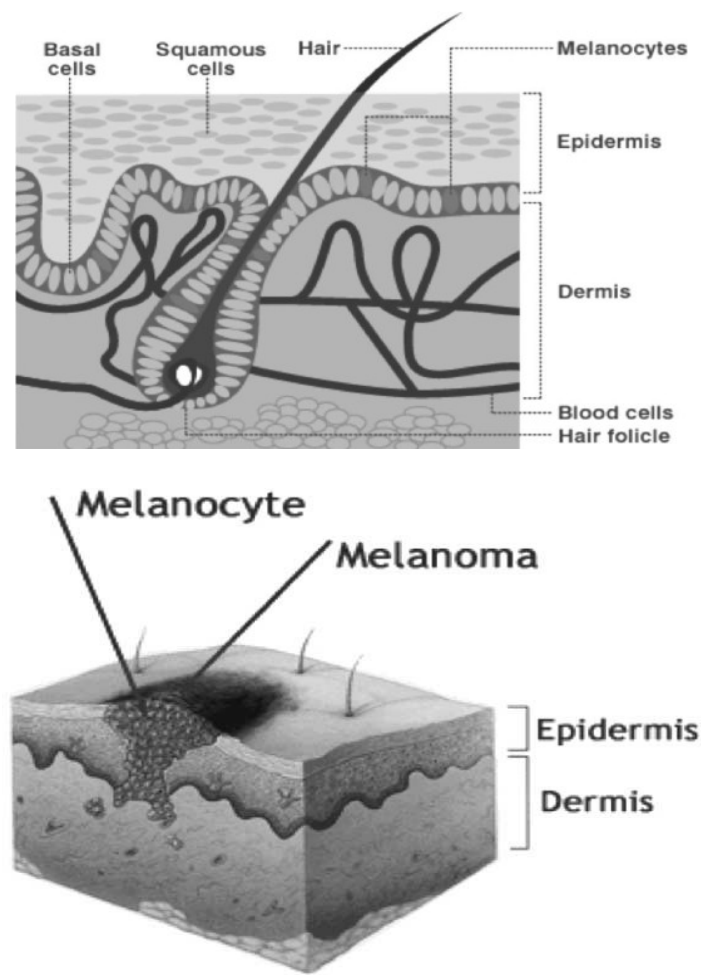

Figure 1 : skin 
Types of Skin Cancer :

The common types are:

- Melanoma: Melanoma begins in melanocytes. Melanoma can occur on skin. Melanoma is found in dark skin people.

- Basal cell skin cancer: Basal cell skin cancer begins in the basal cell layer of the skin. It occurs in face. Basal cell skin cancer is the most common type of skin cancer.

- Squamous cell skin cancer: Squamous cell skin cancer begins in squamous cells. squamous cell skin cancer is the most common type of skin cancer found in dark people.

This paper is simply implemented algorithms for detection of skin cancer and also finding relative area, which have steps : Preprocessing by using median filter[2][3]. Median filter is removed unwanted hair, bubbles. The skin cancer images usually contains fine hair, noise and air bubbles. These are not cancer factor, which we want to remove by using median filter. Second step is segmentation by Fuzzy C-Means. Stage of segmentation is FCM and FCM is a technique for establishing boundaries in image. Third step is feature extraction done by Gray Level Co-occurance Matricx[6].

\section{Methodology}

This section describes our flow of algorithm or steps. The main algorithm is shown in figure. Each block its own algorithm operation.

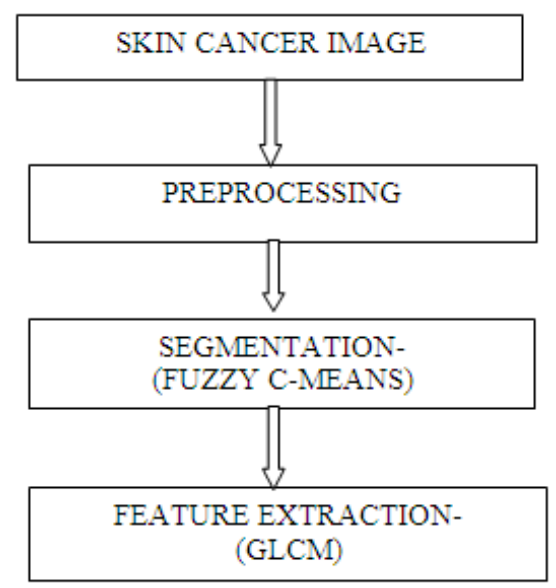

Figure 2: Flow of proposed algorithm

A. $\quad$ Image preprocessing :

Preprocessing is to perform image processing on 1 image by using median filter to obtain enhanced image. The median operator is a nonlinear filter. A median filter make smoothers the skin cancer image. The concept of a median filter was introduced by Tukey in 1977. Its extension to two-dimensional images was discussed by Pratt in 1978.

The median filter reduces the variance of the intensities in the image. Median filter preserve skin cancer image edge shapes.

Median filter perform the following tasks to find each pixel value in the processed image.

1. The values of the pixels in the neighborhood are placed into an array.

2. The values in the array are then sorted in the ascending (or) descending order.

3. The value in the center of the sorted list is placed into the original centeral pixel location in a new image.

4. Then the neighborhood is shifted over to the next pixel and the process is repeated.

\section{B. Segmentation:}

Segmentation is one of the important steps in cancer detection. The aim of segmentation process is to divide the image into homogeneous, self-consistent regions, which should correspond to different objects in the scene. The process is achived using only properties of image. The properties are edges and texture are useful for segmentation. Image segmentation is the process of image partitioning into groups of pixels which are homogenous with respect to some criterion. Segmentation is concerned with dividing an image into meaningful regions.

Skin cancer image segmentation is based on thresholding [4][5]. It is an example of pixel based segmentation algorithm. Thresholding techniques produce segments having pixels with similar intensities. Thresholding is a technique for establishing boundaries in images that contain solid objects resting on contrasting background. There exists a large segmentation method. Another method for segmentation is fuzzy C- 
Means[6][7]. Fuzzy C-Means is an unsupervised clustering algorithm that has been applied to problems involving clustering feature analysis. FCM has more applications such as agricultural engineering, astronomy, geology, image analysis, medical diagnosis, shape analysis, chemistry. Fuzzy C-Means segmentations is based on partial clustering. Fuzzy C-Means is more flexible.

Algorithm for Fuzzy C-Means

1. Initialize the cluster center.

2. Initialize the fuzzy partition membership function.

3. Again compute new cluster center.

4. Repeat step 2 to 3.

In the skin cancer image, cancer is clearly seen by using median filter. Thus, we can divide segmentation image into two parts object and background part.

\section{Feature Extraction :}

There are some important features that distinguish skin cancer. Feature extraction extracts some important features of image data from the segmentation image. It makes the raw data more useful in prossesing. By feature extraction, the image data is narrow down to a set of features. Feature extraction technique used is Gray Level Co-occurrence Matrix (GLCM)[6]. The GLCM is a powerful tool for image feature extraction by mapping the gray level co-occurrence probabilities based on spatial relations of pixels in different angular directions. The feature extracted based on GLCM are: Contrast, Correlation, Energy, and Homogeneity.Contrast is the measure ofcontrast or local intensity variation of pixel. Correlation is a measure of gray level linear dependence between the pixel at specified positions relative to each other. A homogeneous scene will contain only a few gray levels. It provides GLCM with only a few but relatively high values. Energy is returns the sum of squared elements in the GLCM. The idea is to calculate the co-occurrence matrix to find statistic values, for instance homogeneity, contrast,correlation and energy.

Table 1 Statistic Properties

\begin{tabular}{|c|c|c|}
\hline Property & Description & Formula \\
\hline 'Contrast' & $\begin{array}{l}\text { Returns a measure of the intensity contrast between a pixel and its } \\
\text { neighbor over the whole image. } \\
\text { Range }=\left[0(\text { size }(\text { GLCM, } 1)-1)^{\wedge} 2\right] \\
\text { Contrast is } 0 \text { for a constant image. }\end{array}$ & $\sum_{i, j}|i-j|^{2} p(i, j)$ \\
\hline 'Correlation' & $\begin{array}{l}\text { Returns a measure of how correlated a pixel is to its neighbor over the } \\
\text { whole image. } \\
\text { Range }=[-11] \\
\text { Correlation is } 1 \text { or }-1 \text { for a perfectly positively or negatively correlated } \\
\text { image. Correlation is NaN for a constant image. }\end{array}$ & $\sum_{i, j} \frac{(i-\mu i)(j-\mu j) p(i, j}{\sigma_{i} \sigma_{j}}$ \\
\hline 'Energy' & $\begin{array}{l}\text { Returns the sum of squared elements in the GLCM. } \\
\text { Range }=\left[\begin{array}{ll}0 & 1\end{array}\right] \\
\text { Energy is } 1 \text { for a constant image. }\end{array}$ & $\sum_{i, j} p(i, j)^{2}$ \\
\hline 'Homogeneity & $\begin{array}{l}\text { Returns a value that measures the closeness of the distribution of } \\
\text { elements in the GLCM to the GLCM diagonal. } \\
\text { Range }=\left[\begin{array}{ll}0 & 1\end{array}\right] \\
\text { Homogeneity is } 1 \text { for a diagonal GLCM. }\end{array}$ & $\sum_{i, j} \frac{p(i, j)}{1+|i-j|}$ \\
\hline
\end{tabular}

\section{Results}

Here software MATLAB 10 version is used for algorithm. We take some sample image of skin cancer by National Cancer Institute. By using, sample images we can detect the skin cancer and also detect the shape of cancer. Some images results are shown in figure. 


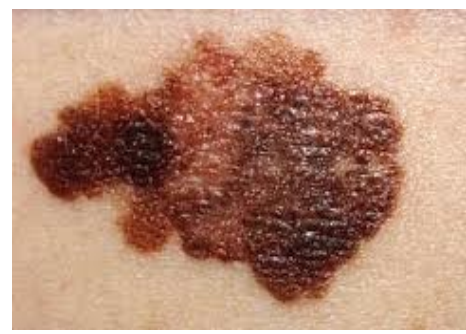

(a)

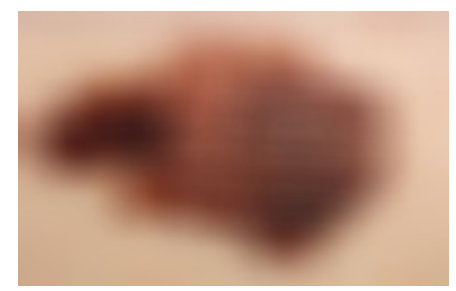

(b)

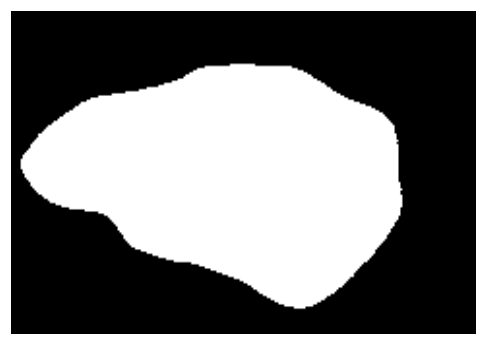

(c)

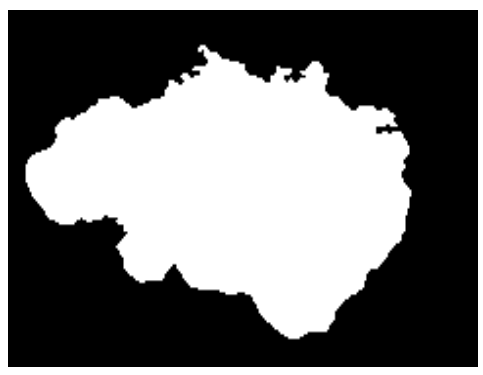

(d)

Figure 3 :(a) Original image

(b) Preprocessed image

(c)Thresholding segmentation

(d) FCM Segmentation

Table 2. Feature Extraction

\begin{tabular}{|c|c|c|c|c|c|c|}
\hline \multirow{2}{*}{ Image } & Relativ & \multicolumn{4}{|c|}{ GLCM } & $\begin{array}{c}\text { Standard } \\
\text { e area }\end{array}$ \\
\cline { 3 - 6 } & $\begin{array}{c}\text { contr } \\
\text { ast }\end{array}$ & $\begin{array}{c}\text { correl } \\
\text { ation }\end{array}$ & $\begin{array}{c}\text { ener } \\
\text { gy }\end{array}$ & $\begin{array}{c}\text { homo } \\
\text { geneit } \\
\mathbf{y}\end{array}$ & $\begin{array}{c}\text { contour } \\
\text { signature }\end{array}$ \\
\hline $\begin{array}{c}\text { Figure } \\
\text { 3(a) }\end{array}$ & $\begin{array}{c}41.527 \\
8\end{array}$ & $\begin{array}{c}0.274 \\
3\end{array}$ & $\begin{array}{c}0.919 \\
2\end{array}$ & 0.36 & 0.918 & 16.2734 \\
\hline
\end{tabular}

\section{Conclusion}

The segmentation method presented in this paper is one of the flexibly methods for segmentation. Thresholding segmentation is useful technique for establishing boundaries in image. The results obtained in segmentation images are taken by national cancer institute[1]. In this case also we find infected area.Cancerous region is separated from healthy skin by the method of segmentation. The unique features of the segmented images were extracted using GLCM. Based on the features, the image can be classified. 


\section{References}

[1] Www.national cancer institute

[2] Sookpotharom Supot, "Border Detection of Skin Lession Images Based on Fuzzy C-Means Thresholding," in Third International Conference on Genetic and Evolutionary Computing, pp. 777-780 , 2009

[3] Azaden Noori Hoshyar, Adel Al-Jumaily, and Riza Sulaiman,"Review On Automatic Early skin Cancer Detection," in International Conference in Computer science and Service System(CSSS), pp.4036-4039, 2011.

[4] Margarida Silveria, Jacinto C. Nascimento, Jorge S. Marques, Andre R.S.Marcal, Teresa Mendonca, Syogo Yamauchi, Junji Maeda, and Jorge Rozeira, "Comparison of Segmentation Methods For Melanoma Diagnosis in Dermoscopy Images," IEEE Journal of Selected Topic in Signal Processing, vol.3, no.1, pp. 35-45, February 2009.

[5] Dr. J. Abdul Jaleel, Sibi Salim, and Aswin R.B, “Artificial Neural Network Based Detection of Skin Cancer,” International Journal of Advanced Research in Electronics and Instrumentation Engineering, vol.1, no. 3, September 2012.

[6] J.Abdul Jaleel, Sibi Salim, and Aswin.R.B, “ Computer Aided Detection of Skin Cancer," IEEE Conference on Circuits, Power and Computing Technologies. pp 1137-1142,2013.

[7] Ilias Maglogiannis, Member, IEEE, and Charalampos N. Doukas, Student Member, IEEE,”Overview of Advanced Computer Vision Systems for Skin Lesions Characterization," transactions on information technology in biomedicine, Vol 13,pp 721733,2009 . 